\title{
On the use of consistent approximations for the optimal design of beams*
}

\author{
C. Kirjner-Neto and E. Polak \\ Department of Electrical Engineering and Computer Sciences, \\ University of California, Berkeley, CA 94720, USA.
}

\begin{abstract}
We present a strategy, based on the theory of consistent approximations, for solving a class of optimal beam design problems. The paper includes a numerical example.

Keywords

Optimal design, discretization theory, consistent approximations
\end{abstract}

\section{INTRODUCTION}

There is a considerable literature dealing with numerical methods for the solution of optimal design problems (see, e.g., Banichuk (1990), Pironneau (1984) and references therein). A major class of current methods constructs an infinite sequence of finite-dimensional approximating problems by means of numerical integration techniques. The theoretical treatment tends to be limited to showing that global minimizers of approximating problems converge to global minimizers of the original problem. The possibility of local minimizers of the approximating problems converging to nonstationary points of the original problem is ignored, and efficient diagonalization techniques (such as those in Polak (1993)) are not explored.

Recently, an abstract theory of consistent approximations for optimization problems has been proposed in Polak (1993). It is based on the concepts of epi-convergence and optimality functions, and provides conditions that ensure convergence of global minimizers, local minimizers and stationary points of the approximating problems to those of the original problem. It also provides diagonalization techniques that make it possible to use efficiently nonlinear programming libraries in solving infinite-dimensional optimization problems.

* The research reported herein was sponsored by the NSF grant ECS 9302926. 
In this paper, we apply the theory of consistent approximations to the solution of a class of optimal Euler-Bernoulli beam design problems with continuum constraints, such as constraints on vertical deflection, and on stresses. We show that the original constraints must be relaxed to ensure that the approximating problems have solutions, and that norm preserving transformations must be used in solving the approximating problems, in order to preserve the conditioning of the original problem with respect to algorithms that are not scale invariant. For ease of exposition we restrict ourselves to beams with rectangular cross section, fixed width, and distributed loads. Extensions to beams of varying width and various cross sections are straightforward. A design example is included.

\section{CONSISTENT APPROXIMATIONS}

Let $\mathcal{B}$ be a topological vector space and consider the problem

$\mathbf{P}$

$$
\min _{z \in Z} f(z)
$$

where $f: \mathcal{B} \rightarrow \mathbb{R}$ is continuous and $Z \subset \mathcal{B}$ is the feasible set. Let $\left\{\mathcal{B}_{N}\right\}_{N=1}^{\infty}$ be a family of finite-dimensional subspaces of $\mathcal{B}$ such that $\mathcal{B}_{N} \subset \mathcal{B}_{N+1}$, for all $N$, and consider the family of approximating problems

$$
\mathbf{P}_{N} \quad \min _{z \in Z_{N}} f_{N}(z), \quad N \in \mathbb{N} \text {, }
$$

where $f_{N}: \mathcal{B}_{N} \rightarrow \mathbb{R}$ is continuous, and $Z_{N} \subset \mathcal{B}_{N}$ (often $Z_{N}=\mathcal{B}_{N} \cap Z$ ).

Definition 2.1 [Attouch (1984)]. The problems in the family $\left\{\mathbf{P}_{N}\right\}_{N=1}^{\infty}$ converge epigraphically to $\mathbf{P},\left(\mathbf{P}_{N} \rightarrow^{E p i} \mathbf{P}\right)$ if : (a) for every $z \in Z$, there exists a sequence $\left\{z_{N}\right\}_{N=1}^{\infty}$, with $z_{N} \in Z_{N}$, such that $z_{N} \rightarrow z$ and $\overline{\lim } f_{N}\left(z_{N}\right) \leq f(z)$; and $(b)$ for every sequence $\left\{z_{N_{k}}\right\}_{k=1}^{\infty}$, with $z_{N_{k}} \in Z_{N_{k}}$, such that $z_{N_{k}} \rightarrow z$ as $k \rightarrow \infty, z \in Z$ and $\underline{\lim } f_{N_{k}}\left(z_{N_{k}}\right) \geq f(z)$.

Definition 2.2. A function $\theta: \mathcal{B} \rightarrow \mathbb{R}\left(\theta_{N}: \mathscr{B}_{N} \rightarrow \mathbb{R}\right)$ is an optimality function for $\mathbf{P}\left(\mathbf{P}_{N}\right)$ if (i) $\theta(\cdot)\left(\theta_{N}(\cdot)\right)$ is sequentially upper semicontinuous, (ii) $\theta(z) \leq 0\left(\theta_{N}(z) \leq 0\right.$ ) for all $z \in \mathcal{B}$ $\left(z \in \mathcal{B}_{\mathcal{X}}\right)$, and (iii) $\theta(\hat{z})=0\left(\theta_{N}(\hat{z})=0\right)$ for any $\hat{z} \in Z$ that is a local minimizer for $\mathbf{P}$ (for any $\hat{z} \in Z_{N}$ that is a local minimizer for $\mathbf{P}_{N}$ ).

Definition 2.3. Let $\theta(\cdot), \theta_{N}(\cdot), N \in \mathbb{N}$, be optimality functions for $\mathbf{P}, \mathbf{P}_{N}$, respectively. The pairs $\left(\mathbf{P}_{N}, \theta_{N}\right)$, in the sequence $\left\{\left(\mathbf{P}_{N}, \theta_{N}\right)\right\}_{N=1}^{\infty}$ are consistent approximations to the pair $(\mathbf{P}, \theta)$, if (i) $\mathbf{P}_{N} \rightarrow{ }^{E p i} \mathbf{P}$, and (ii) for any sequence $\left\{z_{N}\right\}_{N \in K}, K \subset \mathbb{N}$, with $z_{N} \in \mathcal{B}_{N}$ for all $N \in K$, such that $z_{N} \rightarrow z, \varlimsup \lim \theta_{N}\left(z_{N}\right) \leq \theta(z)$.

Theorem 2.4. [Polak (1993)] Suppose that the pairs $\left(\mathbf{P}_{N}, \theta_{N}\right)$ in the sequence $\left\{\left(\mathbf{P}_{N}, \theta_{N}\right)\right\}_{N=1}^{\infty}$ are consistent approximations to the pair $(\mathbf{P}, \theta)$, and that $\left\{\hat{z}_{N}\right\}_{N=1}^{\infty}$ is a sequence such that $\hat{z}_{N} \in Z_{N}$ for all $N$ and $\hat{z}_{N} \rightarrow \hat{z}$. (a) If the $\hat{z}_{N}$ are global minimizers for the $\mathbf{P}_{N}$, then $\hat{z}$ is a global minimizer of $\mathbf{P}$. (b) If $\hat{z}_{N}$ are strict local minimizers whose radii of attraction do not converge to zero, as $N \rightarrow \infty$, then $\hat{z}$ is a local minimizer of $\mathbf{P}$. (c) If $\overline{\lim } \theta_{N}\left(\hat{z}_{N}\right)=0$, then $\theta(\hat{z})=0$.

\section{CONSISTENT APPROXIMATIONS FOR A FIXED BEAM DESIGN}


Beam model Consider a fixed Euler-Bernoulli beam with modulus of elasticity $E>0$, length $L>0$, rectangular cross-section, constant width $b>0$, and variable depth defined by a positive, Lipschitz continuous function $h:[0, L] \rightarrow \mathbb{R}$. The beam is subjected to a vertical load with density $l(h, \cdot)$ of the form

$l(h, x)=m(x)-K h(x), \quad x \in[0, L]$,

where $K \geq 0$ is a constant, and $m(\cdot)$ is piecewise Lipschitz continuous, with finitely many points of discontinuity in $[0, L]$.

For a cantilever beam of length $L$, depth $h(x)$, and subject to the load density $l(h, \cdot)$, the bending moment, $M_{c}(h, \cdot)$, is the unique solution of the final value problem

$M_{c}^{\prime \prime}(h, x)=l(h, x), x \in[0, L], M_{c}(h, L)=M_{c}^{\prime}(h, L)=0$.

The bending moment, $M(h,-)$, in a fixed beam of length $L$, depth $h(x)$, and load density $l(h, \cdot)$, differs from $M_{c}(h, \cdot)$ only by an affine term in $x$, which is due to the reactions at the support points. Therefore

$M(h, x)=M_{c}(h, x)+g_{1}(h) x+g_{2}(h), \quad x \in[0, L]$.

It follows from the dual formulation of the variational problem associated with the bending of the fixed beam (see Reedy (1984)), that $g(h) \triangleq\left[g_{1}(h) g_{2}(h)\right]^{T}$ satisfies the equation

$\left[\begin{array}{cc}\int_{0}^{L} \frac{x^{2}}{h(x)^{3}} d x & \int_{0}^{L} \frac{x}{h(x)^{3}} d x \\ \int_{0}^{L} \frac{x}{h(x)^{3}} d x & \int_{0}^{L} \frac{1}{h(x)^{3}} d x\end{array}\right]\left[\begin{array}{l}g_{1}(h) \\ g_{2}(h)\end{array}\right]=\left[\begin{array}{l}-\int_{0}^{L} \frac{M_{c}(h, x) x}{h(x)^{3}} d x \\ -\int_{0}^{L} \frac{M_{c}(h, x)}{h(x)^{3}} d x\end{array}\right]$.

The shear force, $V(h, x)$, and the deflection of the beam, $y(h, \cdot)$, are given by

$V(h, x)=-M^{\prime}(h, x)=-M_{c}^{\prime}(h, x)-g_{1}(h), x \in[0, L]$,

$y^{\prime \prime}(h, x)=12 M(h, x) / E b h(x)^{3}, x \in[0, L], y(h, 0)=y^{\prime}(h, 0)=0$.

For design purposes we assume that the depth function is an element of the set

$H_{a d} \triangleq\{h \in C[0, L]|0<\alpha \leq h(x) \leq \beta| d h,(x) / d x \mid \leq \gamma$, for a. e. $x \in[0, L]\}$,

where $0<\alpha<\beta<\infty$ and $\gamma \geq 0$ are given constants, and $C[0, L]$ is the space of continuous real-valued functions defined on $[0, L]$.

The "natural" norm on $C[0, L]$ for establishing continuity and differentiability of solutions of $(3.2 \mathrm{a}-\mathrm{e})$ with respect to depth functions $h(\cdot)$ is the sup-norm, $\| \boldsymbol{l}_{\infty}$, while the "natural" norm for defining optimality functions is the $L_{2}[0, L]$ norm, $\mid \cdot l_{2}$. Hence, we will work in the "compromise" inner-product space $\left(C[0, L],|\cdot|_{2},\langle\cdot, \cdot\rangle_{2}\right)$, where $\langle\cdot, \cdot\rangle_{2}$ denotes the usual inner-product on $L_{2}[0, L]$. As a consequence, we have to use the concepts of continuity and differentiability relative to $H_{a d}$.

Definition 3.1 Let $\left(V, \| \cdot I_{V}\right)$ be a normed space and let $\zeta$ be a function from $H_{a d}$ into $V$.

(a) $\zeta(\cdot)$ is Lipschitz continuous relative to $H_{a d}$ if there exists an $C<\infty$, such that for all $h, h^{\prime} \in H_{a d},\left\|\zeta(h)-\zeta\left(h^{\prime}\right)\right\|_{V} \leq C \| h-h^{\prime} \mathbf{l}_{2}$. 
(b) $\zeta(\cdot)$ is differentiable relative to $H_{a d}$ if for any $h \in H_{a d}$ there exists a continuous linear map $D \zeta(h ; \cdot)$, called the $H_{a d}$-derivative of $\zeta(\cdot)$ at $h$, such that

$\lim _{h^{\prime} \in H_{a d}, \mathbf{l}^{\prime}-h \mathbf{I}_{2} \rightarrow 0} \mid \zeta\left(h^{\prime}\right)-\zeta(h)-D \zeta\left(h ; h^{\prime}-h\right) \|_{V} N h^{\prime}-h \mathbf{I}_{2}=0$.

Theorem 3.2. The mappings $h \mapsto M(h, \cdot), h \mapsto V(h, \cdot)$, and $h \mapsto y(h, \cdot)$, from $H_{a d}$ into $\left(C[0, L],\|\cdot\|_{\infty}\right)$, are all Lipschitz continuously differentiable relative to $H_{a d}$.

We will denote the $H_{a d}$-derivatives at $h$, of the mappings introduced above, by $D_{1} M(h, \cdot ; \cdot), D_{1} V(h, \cdot ; \cdot)$, and $D_{1} y(h, \cdot \cdot \cdot)$, respectively.

Problem formulation We will consider optimal beam design problems of the form

$$
\mathbf{P} \quad \min _{h \in H_{o d}}\left\{f(h) \mid \psi(h) \triangleq \max _{j \in \mathbf{q}} \max _{x \in[0, L]} \phi^{j}(h, x)-r^{j}(x) \leq 0\right\} \text {, }
$$

where for any $q>0, \mathbf{q} \triangleq\{1, \ldots, q\}$, and $f(\cdot)$ and $\phi^{j}(\cdot, \cdot), j \in \mathbf{q}$, have the form

$f(h)=\int_{0}^{L} \phi^{0}(h, x) d x$,

$\phi^{j}(h, x)=\tilde{\phi}^{j}(h(x), M(h, x), V(h, x), y(h, x), x), j \in \overline{\mathbf{q}}$,

with $M(h, \cdot), V(h, \cdot)$, and $y(h, \cdot)$ determined by $(3.2 \mathrm{~b}-\mathrm{f})$, and for $j \in \overline{\mathbf{q}} \triangleq\{0,1, \ldots, q\}$, $\bar{\phi}^{j}:[\alpha, \beta] \times \mathbb{R} \times \mathbb{R} \times \mathbb{R} \times[0, L] \rightarrow \mathbb{R}$.

Assumption 3.3. (a) The functions $r^{j}(\cdot), j \in q$, are Lipschitz continuously differentiable on $[0, L]$ and satisfy

$\min _{j \in \mathbf{q}} \min _{x \in[0, L]} r^{j}(x)=\hat{r}>0$.

(b) The functions $\tilde{\phi}^{j}(\cdot, \cdot, \cdot, \cdot, \cdot), j \in \overline{\mathbf{q}}$, are Lipschitz continuously differentiable.

(c) The feasible set for $\mathbf{P}$ is non-empty.

It follows from (3.5b-c), the Lipschitz continuous differentiability of $\widetilde{\phi}^{j}(\cdot, \cdot, \cdot, \cdot, \cdot)$, $j \in \overline{\mathbf{q}}$, and Theorem 3.2, that $h \mapsto \phi^{j}(h, \cdot), j \in \overline{\mathbf{q}}$, and $h \mapsto f(h)$ are Lipschitz continuously differentiable functions relative to $H_{a d}$. We will denote by $D_{1} \phi^{j}(h, \cdot ;), j \in \overline{\mathbf{q}}$, and Df $(h ; \cdot)$ their $H_{a d}$-derivatives.

Following Polak (1987), we we define the optimality function $\theta: H_{a d} \rightarrow \mathbb{R}_{-}$, for $\mathbf{P}$, by

$\theta(h) \triangleq \min _{h^{\prime} \in H_{a d}} \tilde{F}\left(h, h^{\prime}\right)$,

where $\psi(h)_{+} \triangleq \max \{\psi(h), 0\}$, and

$\tilde{F}\left(h, h^{\prime}\right) \triangleq \max \left\{D f\left(h ; h^{\prime}-h\right)-\psi(h)_{+}, \max _{j \in \mathbf{q}} \max _{x \in[0, L]} \phi^{j}(h, x)+D_{1} \phi^{j}\left(h, x ; h^{\prime}-h\right)\right.$

$$
\left.-\psi(h)_{+}\right\}+1 / 2 \| h^{\prime}-h \mathbf{I}_{2}^{2} \text {. }
$$

Theorem 3.4. (a) $\theta: H_{a d} \rightarrow \mathbb{R}$ is upper semicontinuous; (b) If $\hat{h}$ is a local minimizer of P, then $\theta(\hat{h})=0$; (c) For any $\hat{h} \in H_{a d}$ such that $\psi(\hat{h}) \leq 0, \theta(\hat{h})=0$ if and only if $d F(\hat{h} ; h-\hat{h}) \geq 0$, for all $h \in H_{c d d}$, where $\left.F(h) \triangleq \max \left\{f(h)-f^{\gamma}\right), \psi(h)\right\}$.

Discretization Following the theory in Section 2, we select a family of finitedimensional subspaces of $C[0, L]$, specified by basis sets. For every integer $N>0$ we let $\Delta_{N} \triangleq L / N$ and define $x_{N, k} \triangleq(k-1) \Delta_{N}, k \in \mathbf{N}+\mathbf{1}$. Let 


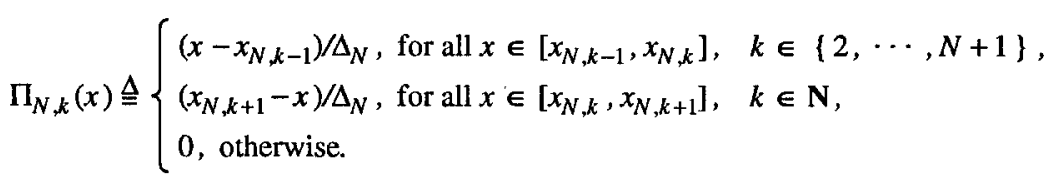

Let by $H_{N}$ the span of the basis set $\left\{\Pi_{N, k}\right\}_{k=1}^{N+1}$ and let $H_{a d, N} \triangleq H_{N} \cap H_{a d}$.

Next, after transcription into first order form, we use Euler's method to discretize the initial value problem (3.2e) and the final value problem (3.2a), and the rectangle rule to approximate the integrals in (3.2c). It is not at all clear that using a higher order integration scheme would be more efficient in optimal design.

Let $M_{N}\left(h, x_{N, k}\right), V_{N}\left(h, x_{N, k}\right)$, and $y_{N}\left(h, x_{N, k}\right), k \in \mathbf{N}+1$, denote the discretized bending moment, shear force, and deflection, respectively. The following result is a direct consequence of the Implicit Function Theorem.

Lemma 3.5. The maps $h \mapsto M_{N}(h, \cdot), h \mapsto V_{N}(h, \cdot)$, and $h \mapsto y_{N}(h, \cdot)$, from $H_{a d, N}$ into $\mathbb{R}^{N+1}$, are Lipschitz continuous differentiable.

Approximating problems. We now define the family of approximating problems $\mathbf{P}_{N}, N=1,2, \ldots$, as follows:

$\mathbf{P}_{N} \min _{h \in H_{a d, N}}\left\{f_{N}(h) \mid \psi_{N}(h) \triangleq \max _{j \in \mathbf{q}} \max _{k \in N+1} \phi_{N}^{j}\left(h, x_{N, k}\right)-\left(1+\Delta_{N}^{1 / 2}\right) r^{j}\left(x_{N, k}\right) \leq 0\right\}$,

where

$f_{N}(h) \triangleq \sum_{k=1}^{N} \phi_{N}^{0}\left(h, x_{N, k}\right) \Delta_{N}$,

$\phi \dot{N}\left(h, x_{N, k}\right)=\tilde{\phi}^{j}\left(h\left(x_{N, k}\right), M_{N}\left(h, x_{N, k}\right), V_{N}\left(h, x_{N, k}\right), y_{N}\left(h, x_{N, k}\right), x_{N, k}\right), j \in \overline{\mathbf{q}}$.

The term $\Delta_{N}^{1 / 2}$ in (3.9a) is added to guarantee that for $N$ large enough the feasible set for $\mathbf{P}_{N}$ is non-empty, a fact needed in the proof of Theorem 3.7 (a) below.

Equation (3.9c) defines the functions $\phi_{N}^{j}(h, \cdot), j \in \overline{\mathbf{q}}$, only on the mesh points $x_{N, k}$, $k \in \mathbf{N}+1$. We define $\phi \dot{X}(h, \cdot):[0, L] \rightarrow \mathbb{R}$ as the piecewise affine interpolation of the values $\phi \dot{K}\left(h, x_{N, k}\right), k \in \mathbf{N}+1$. It follows from (3.9c) and Assumption 3.3(b), that the $\phi_{N}^{j}(\cdot, \cdot), j \in \overline{\mathbf{q}}$, are Lipschitz continuously differentiable on $H_{a d, N}$. The derivatives of the mappings $h \mapsto \phi_{\mathcal{N}}(h, \cdot), j \in \overline{\mathbf{q}}$, and $h \mapsto f(h)$, denoted by $D_{1} \phi_{\mathcal{N}}(h, \cdot ; \cdot)$ and $D f_{N}(h ; \cdot)$, can be obtained using (3.9b,c), Lemma 3.5 and the Chain Rule.

Next, we define the optimality functions $\theta_{N}: H_{a d, N} \rightarrow \mathbb{R}$ by

$\theta_{N}(h) \triangleq \min _{h^{\prime} \in H_{\alpha d, N}} \tilde{F}_{N}\left(h, h^{\prime}\right)$,

where $\tilde{F}_{N}(\cdot, \cdot)$ is defined as in $(3.7 \mathrm{~b})$, with all the functions modified by the addition of the subscript $N$. Results analogous to Theorem 3.4 hold for $\theta_{N}\left({ }^{\circ}\right)$. Also note that one can evaluate $\theta_{N}(\cdot)$ by solving a positive definite quadratic program.

Consistency of Approximations The following is proved in Kirjner-Neto (1994).

Lemma 3.6. (a) For each $h \in H_{a d}$, and $N \geq 1$, there exists $h_{N} \in H_{a d, N}$ such that

$\max _{x \in[0, L]}\left|h(x)-h_{N}(x)\right| \leq \gamma \Delta_{N}$.

(b) There exists a $C \in \mathbb{R}$ such that for all $j \in \overline{\mathbf{q}}, N \geq 1, h \in H_{a d}$, and $h_{N} \in H_{a d, N}$, 


$$
\begin{aligned}
& \max _{x \in[0, L]}\left|\phi^{j}(h, x)-\phi \dot{N}\left(h_{N}, x\right)\right| \leq C\left[\Delta_{N}+\left\|h-h_{N}\right\|_{2}\right], \\
& \left|\psi(h)-\psi_{N}\left(h_{N}\right)\right| \leq C\left[\Delta_{N}^{1 / 2}+\mid h-h_{N} \|_{2}\right], \\
& \left|f(h)-f_{N}\left(h_{N}\right)\right| \leq C\left[\Delta_{N}+\mid h-h_{N} !_{2}\right] .
\end{aligned}
$$

In view of the definitions of $\psi(h)$ and $\psi_{N}(h)$, it is clear that the feasible sets $\mathbf{Z}$ and $\mathbf{Z}_{N}$ for $\mathbf{P}$ and $\mathbf{P}_{N}$ satisfy: $\mathbf{Z}=\left\{h \in H_{a d} \mid \psi(h) \leq 0\right\}$, and $\mathbf{Z}_{N}=\left\{h \in H_{a d, N} \mid \Psi_{N}(h) \leq 0\right\}$.

Theorem 3.7. (Epiconvergence) (a) For every $h \in \mathbf{Z}$, there exists a sequence $\left\{h_{N}\right\}_{N=N_{0}}^{\infty}$, with $h_{N} \in \mathbf{Z}_{N}$, such that $f_{N}\left(h_{N}\right) \rightarrow f(h)$ as $N \rightarrow \infty$. (b) Let $\left\{h_{N}\right\}_{N=N_{0}}^{\infty}$ be a sequence such that $h_{N} \in \mathbf{Z}_{N}$ and $h_{N} \rightarrow \hat{h}$ as $N \rightarrow \infty$, then $\hat{h} \in \mathbf{Z}$, and $f_{N}\left(h_{N}\right) \rightarrow f(\hat{h})$.

Proof. Suppose $h \in \mathbf{Z}$ is given. Then, by Lemma 3.9 (a), for each integer $N$, there exists an $h_{N} \in H_{a d, N}$ such that (3.11a) holds. Clearly, $h_{N} \rightarrow h$ as $N \rightarrow \infty$. It follows from (3.11d) that $f_{N}\left(h_{N}\right) \rightarrow f(h)$ as $N \rightarrow \infty$. To complete the proof of part $(a)$ it remains to show that there exists an $N_{0}$ such that $h_{N} \in \mathbf{Z}_{N}$ for all $N \geq N_{0}$. Indeed, since $h \in \mathbf{Z}$ by assumption we have $\psi(h) \leq 0$. Hence, using (3.11b) and (3.11a) we obtain

$$
\begin{aligned}
\Psi_{N}\left(h_{N}\right) & \leq \Psi_{N}\left(h_{N}\right)-\psi(h) \\
& =\max _{j \in \mathbf{q}} \max _{x \in[0, L]}\left[\phi \dot{j}\left(h_{N}, x\right)-r^{j}(x)\left(1+\Delta_{N}^{1 / 2}\right)\right]-\max _{j \in \mathbf{q}} \max _{x \in[0, L]}\left[\phi^{j}(h, x)-r^{j}(x)\right] \\
& \leq \max _{j \in \mathbf{q}} \max _{x \in[0, L]}\left[\left|\phi \dot{j}_{N}\left(h_{N}, x\right)-\phi^{j}(h, x)\right|-r^{j}(x) \Delta_{N}^{1 / 2}\right] \\
& \leq C\left[\Delta_{N}+\mid h-h_{N} \|_{2}\right]-\hat{r} \Delta_{N}^{1 / 2} \leq C\left[\Delta_{N}+\left\|h-h_{N}\right\|_{\infty}\right]-\hat{r} \Delta_{N}^{1 / 2} \\
& \leq C(1+\gamma) \Delta_{N}-\hat{r} \Delta_{N}^{1 / 2},
\end{aligned}
$$

where $\hat{r}>0$ is as in (3.6). It follows from (3.12) that there exist an $N_{0}$ such that for all $N \geq N_{0}, \Psi_{N}\left(h_{N}\right) \leq 0$, which proves $(a)$.

Let $\left\{h_{N}\right\}_{N=N_{0}}^{\infty}$ be a sequence as in (b). Since $H_{a d}$ is closed and $\mathrm{Z}_{N} \subset H_{a d, N} \subset H_{a d}$ for all $N \in \mathbb{N}$, it follows that $\hat{h} \in H_{a d}$. The facts that $\hat{h} \in \mathbf{Z}$, that is, that $\psi(\hat{h}) \leq 0$, and that $f_{N}\left(h_{N}\right) \rightarrow f(\hat{h})$ follow directly from (3.11c) and (3.11d) respectively.

Theorem 3.8. Suppose that $\left\{h_{N}\right\}_{N=N_{0}}^{\infty}$, with $h_{N} \in H_{a d, N}$, is such that $h_{N} \rightarrow h$ as $N \rightarrow \infty$. Then $h \in H_{a d}$, and $\overline{\lim }_{N \rightarrow \infty} \theta_{N}\left(h_{N}\right) \leq \theta(h)$.

Corollary 3.9. The sequence $\left\{\left(\mathbf{P}_{N}, \theta_{N}\right)\right\}_{N=1}^{\infty}$ is a family of consistent approximations to the pair $(\mathbf{P}, \theta)$.

\section{TRANSCRIPTION INTO A NONLINEAR PROGRAMMING PROBLEM}

The problems $\mathbf{P}_{N}$ are defined on the finite-dimensional subspaces $H_{N} \subset C[0, L]$. However, numerical computations must be carried out in a finite-dimensional space of coefficients, relative to some basis in $H_{N} \subset C[0, L]$. Note that the basis functions $\Pi_{N, k}(\cdot)$ are not 
orthonormal. Hence, if we define $W_{N}: H_{N} \rightarrow \mathbb{R}^{N+1}$ by $W_{N}(h) \triangleq\left(\eta_{1}, \eta_{2}, \ldots, \eta_{N+1}\right)^{T}$, where $\eta_{k}, k=1, \ldots, N+1$ are the coefficients of $h$ with respect to the basis functions $\Pi_{N, k}$, $k=1, \ldots, N+1$, then we find that $\boldsymbol{l} h\left\|_{2}^{2} \neq \sum_{i=1}^{N+1} \eta_{i}^{2} \triangleq\right\| W_{N}(h) \|^{2}$, i.e., $W_{N}(\cdot)$ is not an isometry. Hence it turns out that if we solve the problem $\mathbf{P}_{N}$, using a nonlinear programming algorithm in this coefficient space, we have inadvertently modified the metric on our original space, which can cause considerable deterioration of performance in non-scale invariant algorithms.

To compute in $\mathbb{R}^{N+1}$, using a metric that is equivalent to the one on $H_{N}$, one can either modify existing nonlinear programming software, something not easily undertaken when using a standard library of programs, or one can solve problems $\overline{\mathbf{P}}_{N}$, equivalent to the $\mathbf{P}_{N}$, and defined in terms of coordinates corresponding to an orthonormal basis.

To define the $\overline{\mathbf{P}}_{N}$ we define $Q_{N} \in \mathbb{R}^{(N+1) \times(N+1)}$ by $\left(Q_{N}\right)_{i j} \triangleq \int_{0}^{L} \Pi_{N, i}(x) \Pi_{N, j}(x) d x$, where for any matrix $A \in \mathbb{R}^{(N+1) \times(N+1)}, A_{i j}$ denotes the $i, j$-th entry of $A$, and define $T_{N}: H_{N} \rightarrow \mathbb{R}^{N+1}$ by $T_{N}(h) \triangleq Q_{N}^{1 / 2} W_{N}(h)$, so that, for any $h \in H_{N}$, and $\vec{h}=T_{N}(h)$, we have

$\|h\|_{2}^{2}=\int_{0}^{L} \sum_{i, j=1}^{N+1}\left(W_{N}(h)\right)_{j} \Pi_{N, j}(x)\left(W_{N}(h)\right)_{i} \Pi_{N, i}(x) d x=\left(Q_{N}^{1 / 2} W_{N}(h)\right)^{T}\left(Q_{N}^{1 / 2} W_{N}(h)\right)=|\bar{h}|^{2}$.

Equation (4.1) implies that the distance between two elements $h$ and $h^{\prime}$ of $H_{N} \subset\left(C[0, L], \mid \cdot \mathbf{I}_{2},\langle\cdot, \cdot\rangle\right)$ is equal to the Euclidean distance between $T_{N}(h)$ and $T_{N}\left(h^{\prime}\right)$.

- Next, let $\bar{H}_{N} \triangleq T_{N}\left(H_{N}\right)$, and let $\bar{H}_{a d, N} \triangleq T_{N}\left(H_{a d, N}\right) \subset \mathbb{R}^{N+1}$. We define the mappings $\bar{f}_{N}: \bar{H}_{a d, N} \rightarrow \mathbb{R}$, and $\phi_{N}\left(\cdot, x_{N, k}\right): \bar{H}_{a d, N} \rightarrow \mathbb{R}, j \in \overline{\mathbf{q}}, k \in \mathbf{N}+\mathbf{1}$ by

$\bar{f}_{N}(\bar{h}) \triangleq \sum_{k=1}^{N} \phi_{N}^{0}\left(T_{N}^{-1}(\bar{h}), x_{N, k}\right) \Delta_{N}, \quad \bar{\phi}_{N}^{j}\left(\bar{h}, x_{N, k}\right) \triangleq \phi_{N}^{j}\left(T_{N}^{-1}(\bar{h}), x_{N, k}\right)$,

which can be computed using (3.9c). Finally, we define the problems $\overline{\mathbf{P}}_{N}, N=1,2, \ldots$, by

$\overline{\mathbf{P}}_{N} \quad \min _{\bar{h} \in \bar{H}_{a d, N}}\left\{\bar{f}_{N}(\bar{h}) \mid \max _{j \in \mathbf{q}} \max _{k \in \mathbf{N}+1} \bar{\phi}_{N}^{j}\left(\bar{h}, x_{N, k}\right)-\left(1+\Delta_{N}^{1 / 2}\right) r^{j}\left(x_{N, k}\right) \leq 0\right\}$,

The following result, establishing equivalence between $\mathbf{P}_{N}$ and $\overline{\mathbf{P}}_{N}, N=1,2, \ldots$, , follows directly from (3.9a-c),(4.2), and (4.3).

Proposition 4.1. (a) $h \in H_{N}$ is feasible for $\mathbf{P}_{N}$ if and only if $\bar{h}=T_{N}(h) \in \mathbb{R}^{N+1}$ is feasible for $\overline{\mathbf{P}}_{N}$, and $(b) h \in H_{N}$ is a global (local) minimizer for $\mathbf{P}_{N}$ if and only if $\bar{h}=T_{N}(h) \in \mathbb{R}^{N+1}$ is a global (local) minimizer for $\overline{\mathbf{P}}_{N}$.

\section{NUMERICAL RESULTS}

To illustrate the use of consistent approximations together with a diagonalization strategy (see Polak (1993)), we solved a problem of the form $P$, with $E=10^{7} \mathrm{psi}, L=50$ in, $b=5$ in, $K=0$, $\alpha=1.0 \mathrm{in}, \beta=5.0 \mathrm{in}$, and $\gamma=0.15$, and continuum constraints on the maximum normal stress $\sigma_{\max }(h, x)$, the maximum shear $\tau_{\max }(h, x)$, and the deflection $y(h, x)$, as follows,

$\left|\sigma_{\max }(h, x)\right| \leq 30,000 \mathrm{psi},\left|\tau_{\max }(h, x)\right| \leq 15,000 \mathrm{psi},|y(h, x)| \leq 0.1 \mathrm{in}$,

for all $x \in[0,1]$. We used the cost function given by $f(h)=\int_{0}^{L} h(x) d x$. The load applied to the beam was $l(x)=-1500 \mathrm{psi}, x \in[20,30], l(x)=0$ otherwise.

The initial discretization was $N=8$ points, and the initial beam depth $h(x) \equiv 2.85$ in (see Figure 1.(a)). The initial cost was 142.5. Figure 1(b) shows the final design obtained in 16 


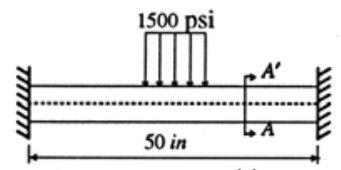

(a)

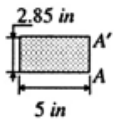

5 in

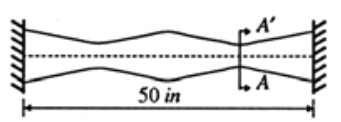

(b)

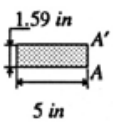

5 in

Fig. 1. (a) Initial Design; (b) Final Design.
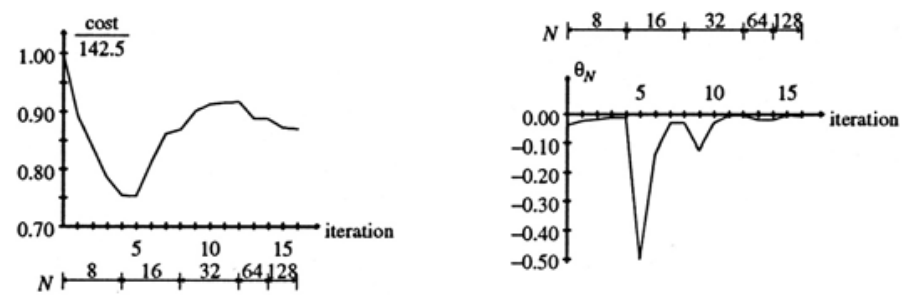

Fig. 2. Computed cost and computed optimality function

iterations of the algorithm in Polak (1991). The final discretization parameter was $N=128$ and cost was 124.05. In Figure 2, we find the cost as a percentage of the initial cost, the value of the optimality function $\theta_{N}$, and the number of discretization points used at each iteration.

\section{REFERENCES}

Attouch, H. (1984) Variational convergence for functions and operators, Pitman.

Banichuk, N. V. (1990) Introduction to optimization of structures, Springer Verlag.

Kirjner-Neto, C. and Polak E. (1994) On the use of consistent approximations for the optimal design of beams, University of California, Berkeley, Memo UCB/ERL M94/22.

Pironneau, O. (1984) Optimal shape design for elliptic systems, Springer-Verlag.

Polak, E. (1987) On the mathematical foundations of nondifferentiable optimization in engineering design, SIAM Review, 29, 21 -91.

Polak, E. (1993) On the use of consistent approximations in the solution of semi-infinite optimization and optimal control problems, Mathematical Programming, B-62, 385-414.

Polak, E. and He, L. (1991) A Unified steerable phase I-Phase II method of feasible directions for semi-infinite optimization, JOTA, 69, 83-107.

Reedy, J. N. (1984) Energy and variational methods in applied mechanics, Wiley \& Sons. 